\title{
Non-Factive Understanding: A Statement and Defense
}

\author{
Yannick Doyle, Spencer Egan, Noah Graham, Kareem Khalifa
}

Forthcoming in the special issue of Journal for General Philosophy of Science on

Normative and Naturalistic Approaches to Understanding (edited by Henk de Regt and Jan Faye)

\section{Introduction}

In epistemology and philosophy of science, there has been substantial debate about truth's relation to understanding. "Non-factivists" hold that radical departures from the truth are not always barriers to understanding; so-called "quasi-factivists" demur. Arguably, the frontline of this debate concerns the proper interpretation of the ideal gas law, with non-factivists (Elgin 2004, 2007, 2009a, b), quasi-factivists (Greco 2013, Kvanvig 2009, Mizrahi 2012), and intermediate positions (Strevens 2008, 2013, 2016) all laying claim to the understanding it provides. Yet these discussions have suffered from confusions about the relevant science, as well as from conceptual confusions. Consistent with the themes of this issue, the proper corrective is both naturalistic and normative: better descriptions of the science, and more careful analytical work.

To that end, we shall argue that the ideal gas law is best interpreted as favoring non-factivism about understanding, but only after delving a bit deeper into the statistical mechanics that has informed these arguments and stating more precisely what non-factivism entails. Section 2 defines non-factivism about understanding in terms of three conditions. Sections 3 through 5 
then show how a statistical-mechanical derivation of the ideal gas law satisfies those three conditions. Along the way, we indicate where earlier discussions have gone astray.

\section{What is Non-Factivism?}

We define non-factivism about understanding as follows:

Non-Factivism: There exist some $p$ and $x$ such that:

(1) $\quad p$ is false;

(2) $\quad p$ provides understanding of $x$; and

(3) The understanding of $x$ resulting from either not accepting $p$ or accepting a more accurate proposition instead of $p$ is not better than the understanding provided by accepting $p$.

Call the first the Falsehood Condition; the second, the Provision Condition, and the third, the Parity Condition. We shall discuss how a statistical-mechanical derivation of the ideal gas law satisfies each of these conditions, and how our formulations of these conditions are an improvement over analogues in the current literature on non-factive understanding.

Before doing so, let us clarify a few general points about our characterization of nonfactivism. First, for our purposes, it does not matter if $p$ is regarded as a single, false proposition that provides understanding of $x$ only in concert with other true propositions, or if $p$ is regarded as a larger conjunction of propositions that is sufficient to provide understanding of $x$ unto itself, but that is nevertheless false because one of its conjuncts is false.

Additionally, we will say that $x$ refers to the object of understanding (i.e. the thing to be understood), and $p$ refers to the basis of understanding (i.e. the thing that provides understanding 
of the object). ${ }^{1}$ We assume that objects come in many forms, e.g. propositions, entities, systems, behaviors, subject matters, and embedded questions. In this way, our discussion applies broadly. Third, while the Provision Condition describes propositions as providing understanding, and the Parity Condition describes understanding as resulting from accepting those propositions, we stipulate that these are two ways of talking about the same relationship. In other words, $p$ provides understanding of $x$ if and only if understanding of $x$ results from accepting that $p$. We further develop these ideas by discussing different ways that propositions provide understanding in Section 4.1 and acceptance's role in understanding in Section 5.2.1.

Fourth, our formulation of non-factivism implies the following as its foil:

Quasi-Factivism: For all $p$ and $x$, if $p$ provides understanding of $x$, then either:

(1) $p$ is true; or

(2) The understanding of $x$ resulting from either not accepting $p$ or accepting a more accurate proposition instead of $p$ is better than the understanding provided by accepting $p$.

As an aside, (strict) factivism is the stronger claim that all bases are true (full stop). To our knowledge, among philosophers of understanding commenting on the ideal gas law, none has endorsed this bold position (see Section 5.) At any rate, as a dialectical point, any argument for non-factivism is an argument against both quasi-factivism and (strict) factivism. Hence, a sound defense of non-factivism exhausts the space of possibilities.

\footnotetext{
${ }^{1}$ Rancourt (forthcoming) coined this distinction.
} 


\section{The Falsehood Condition}

Let us begin with the Falsehood Condition, which requires the basis of understanding to be false. Here, we provide a more detailed account of the derivation of the ideal gas law than has been offered by others working on non-factive understanding. As we shall see below, these details prove vital to adjudicating between non-factivism and quasi-factivism.

While the ideal gas law was first obtained empirically in thermodynamics, today we understand its origin in terms of the statistical mechanics of the underlying microscopic theory. In this approach, the equation of state follows from the partition function $Z$, which is given by a sum over all states of the system in terms of the energy $E$ of each state:

(Eq. 1) $Z=\sum e^{-E / k T}$,

where $k$ is Boltzmann's constant and $T$ is the temperature of the gas. The partition function sums all the states of the system, weighted by their relative probabilities. Roughly stated, the function tells us how many states the system can assume.

Quantities such as energy and pressure can then be derived from this function. For instance, if we assume that the system consists of $N$ identical non-interacting particles, we can factor this sum over states of the whole system into a product of $N$ partition functions for each individual particle. Since the number of states available to each particle scales linearly with volume $V$, each individual partition function will be proportional to $V$. Hence, the full partition function will depend on volume as $V^{N}$, i.e.

(Eq. 2) $\quad Z=V^{N} f(T)^{N} / N !$,

for some function $f(T)$.

For our purposes, (Eq. 2) most important consequence is that the system's (Helmholtz) free energy can be written as the sum of $N \ln V$ plus the terms that are independent of volume. 
Because pressure is proportional to the derivative of free energy with respect to volume, it follows that pressure is inversely proportional to volume. This leads directly to the ideal gas law ${ }^{2}:$

$$
\text { (Eq. 3) } \quad P V=V k T \frac{d \ln Z}{d V}=N k T,
$$

where $P$ denotes the pressure of the gas. We note that $N k$ is equal to $n R$, where $n$ is the number of moles of gas and $R$ is the ideal gas constant.

In our parlance, the ideal gas law is the object of understanding. The assumptions of this derivation constitute the basis of understanding. This example establishes the Falsehood Condition, because the assumption that particles are non-interacting is false (and, as we shall argue below, it provides understanding of the ideal gas law). Indeed, this assumption is not only false, but also internally inconsistent, since some small degree of inter-particle interaction is necessary for the system to reach equilibrium so that quantities such as pressure and temperature are well defined.

\subsection{Two Objections Rebutted}

The Falsehood Condition invites two objections. The first is whether understanding-providing representations must be propositional; the second is whether the idealization, while false, is still approximately true. We address them in turn.

${ }^{2}$ Note that this result holds whether we treat the single particle classically or quantum mechanically. However, for a fully quantum gas, for which two particles have a non-negligible probability of occupying the same state, the need to enforce Bose or Fermi statistics on the full system will introduce an effective interaction among particles, leading to corrections to the ideal gas law. 


\subsubsection{The Non-Propositional Objection}

First, one may object that the Falsehood Condition is needlessly restricted to propositional representations, and to truth and falsehood as the criteria of representational success-a familiar point to those well-versed in the literature on models and scientific representation. However, as the preceding derivation shows, many models are formulated in terms of equations and assumptions which have propositional structure. This suffices for the purpose of establishing non-factivism, even if there are also models which lack this structure. Furthermore, the points below do not hinge on our restriction to propositional representations, and, with a bit of tweaking, could apply more broadly to non-propositional representations.

\subsubsection{The Simple Approximation Objection}

Others have also taken science's use of idealizations as evidence for non-factive understanding (Elgin 2004, 2007, 2009a, b). Quasi-factivists have challenged this argument by claiming that it conflates understanding a model with understanding the phenomena represented by a model (Greco 2013, Kvanvig 2009, Mizrahi 2012, Strevens 2013). For them, it is not the falsehood, particles in an ideal gas do not interact, that provides understanding. Rather, a true proposition describing a more complex relationship between this proposition and reality is the authentic basis of understanding the ideal gas law. If this is right, then it contravenes our claim that the idealization satisfies the Falsehood Condition.

However, quasi-factivists do not say much about this model-reality relationship, nor how propositions about it provide understanding. One elaboration of this idea holds that while a falsehood $p$ does not provide understanding, a "second-order" truth—that " $p$ " is approximately 
true — does (Greco 2013, Mizrahi 2012). ${ }^{3}$ Hence, while the statistical-mechanical model from which the ideal gas law is derived only states that particles do not interact, genuine (or at least better) understanding is achieved so long as this statement is approximately true.

There are two problems with these "simple approximation objections." First, it is not at all obvious that the sentence, "Particles in an ideal gas do not interact," is even approximately true. To see this, consider the other examples of approximations provided by $\operatorname{Greco}(2013,297)$ in his defense of quasi-factivism:

...let $p$ be that it is 3 o'clock. We are happy to say, 'S knows that $p$,' even when $p$ is strictly false, because it is in fact 3:01. Why? Because $p$ is 'true enough.' Similarly for 'You know he never declines an invitation' (when in fact he almost never does) and 'I know the table is level' (when in fact it is not perfectly level).

The first example is the clearest case of an approximation, but there is no obvious analog to 3:00 and 3:01 when discussing particle interactions - it's not as if just a few particles interact in any gas! Similarly, it is not accurate that particles in an ideal gas "almost never" interact, so the analogy with the second example (involving an invitation) is also unclear. Finally, it is not as if there are "perfect interactions," so it is unclear how anything in the idealization could behave like the word "level" in the third example. Closely related, quasi-factivists have not shown how their treatment of idealizations could be regarded as applications of any of the leading accounts

\footnotetext{
${ }^{3}$ It should be noted that, in discussing the ideal gas law, the authors do not distinguish bases from objects in the ways we discuss in Section 4.2.1. For the sake of advancing the discussion, we correct for this ambiguity in this section.
} 
of approximate truth. ${ }^{4}$ Hence, without further elaboration and justification, their claim that idealizations are approximately true is unsupported.

Second, even if idealizations are approximately true, they are strictly false. Hence, simple approximation objections fail to undercut the Falsehood Condition. Lest this point seem like cheap wordplay, it gains force when we consider that less accurate approximations can provide understanding that is at least as good as more accurate representations (see Section 5). For now, we simply note that purveyors of the simple approximation objection have not argued that approximations provide inferior understanding to their strictly true counterparts (or even closer approximations thereof). For instance, if we follow Greco's approach, then the proposition, particles in an ideal gas do not interact, is a close approximation of some strictly true statement $q$ (whatever that may be). However, Greco does not argue that our understanding would improve by replacing the idealization with $q$ in the derivation of the ideal gas law. ${ }^{5}$ Hence, for all that he has shown, a strictly false proposition may provide the greatest understanding of ideal gases.

Therefore, simple approximation objections do not seem to capture the sense in which some idealizations map onto the world, and moreover, do not really address the issue at hand. As such, they pose no threat to the ideal gas law's satisfaction of the Falsehood Condition.

${ }^{4}$ Oddie (2016) reviews the literature on approximate truth.

${ }^{5}$ Insofar as this is a viable option, it would appear that the simple approximation objection collapses into the virial objection, which we discuss in Section 5.1.2. 


\section{The Provision Condition}

Thus far, we have shown that a falsehood figures in the statistical-mechanical derivation of the ideal gas law. But, why should we think that this false assumption provides understanding? This leads us to non-factivism's second requirement, the Provision Condition, which states that accepting a falsehood $p$ provides understanding of $x$.

We begin by describing four ways in which the false assumption of non-interacting particles enhances our understanding of the ideal gas law. We then discuss two ways in which the Provision Condition is an improvement over previous discussions about non-factive understanding.

\subsection{Four Cognitive Goods}

Rather than putting forth a specific account of understanding, let us offer four plausible criteria of understanding, and show that the derivation of the ideal gas law satisfies each of them. In principle, the idealized derivation's satisfaction of just one of these four criteria suffices to show that falsehoods provide understanding. We think it more realistic to hold that these four criteria or "cognitive goods" function as desiderata that are difficult to maximize jointly. Idealizations, then, often strike the "right balance" between these oft-competing desiderata.

\subsubsection{Ease of Calculation}

One cognitive good in the pursuit of understanding is seeing the forest for the trees, i.e. appreciating the deeper structures amidst a larger assemblage of details. A measure of this particular aspect of understanding is the extent to which one avoids getting bogged down in calculations that are only incidental to the phenomenon being understood (De Regt 2009, De Regt and Dieks 2005, Strevens 2008, 2016). For example, if we introduce inter-particle interactions in the partition function for the ideal gas, the factor of $V^{N}$ will be replaced by a 
highly complex sum over all the positions of all the particles, since now the behavior of each particle will be affected by all the other particles in the gas. This will obscure the more invariant statistical-mechanical features that actually underwrite the ideal gas law. Hence, the idealization makes for a tidier derivation, which in turn highlights the main physical factors at work.

\subsubsection{Highlighting Irrelevancies}

Another mark of understanding is the capacity to discriminate information that is relevant to an object's behavior from irrelevant information (Batterman 2000, 2002, Strevens 2008, 2016). Idealizations frequently aid in this task. At low density and high temperature, the interactions of the particles in an ideal gas make no difference to the relations between density, temperature, molarity, and volume. Idealizations flag this irrelevancy by arbitrarily fixing the irrelevant parameters, usually to zero, and showing that the phenomenon of interest (in this case, the ideal gas law) can still be recovered using the other resources of the theory. Thus, representing these interactions as non-existent is an especially vivid way of communicating their irrelevance to the behavior of ideal gases.

\subsubsection{Explanation}

Nearly every theorist of understanding takes grasping an explanation to be sufficient for understanding the explanandum (De Regt 2009, Elgin 2004, Greco 2013, Khalifa 2012, Kvanvig 2003, Strevens 2013). ${ }^{6}$ Plausibly, the derivation above explains why the ideal gas law obtains. If this is correct, then the falsehood, as part of that derivation, provides understanding.

${ }^{6}$ For some nuanced criticisms of this position, see Lipton (2009) and Wilkenfeld and Hellmann (2014). These concerns do not bear on the derivation of the ideal gas law. 
Of course, many find it questionable that a falsehood could figure in an explanation. In particular, sentences such as the following border on the incoherent:

- $\quad$ The patient's bacterial infection explains why she is coughing, but the patient doesn't have a bacterial infection.

- The house burned down because of faulty wiring, but the wiring wasn't faulty.

One might think that these examples support the following general principle:

Explanatory Realism: If " $q$ explains why $p$ " is true or " $p$ because $q$ " is true, then " $q$ " is also true.

We beg to differ. First, at best, explanatory realism may be a good linguistic rule when discussing folk explanations, but need not apply to scientific explanations. After all, only the latter use idealizations with any regularity. Hence, perhaps scientists use the words "explain" or "because" differently than the folk. Indeed, some recent experimental philosophy suggests precisely this difference with respect to the requirement that scientific explanations have true explanantia (Braverman et al. 2012).

Second, the derivation appears to satisfy the core requirements of many prominent models of explanation. For instance, the partition function (Eq. 1) is such that changes to the energy $(E)$ or temperature $(T)$ of the system would result in different equations of state (Eq. 3). Thus, the relationship between the partition function and the ideal gas law exhibits the kind of counterfactual dependence (Lewis 1986, Woodward 2003) or difference-making (Strevens 2008) characteristic of explanation. To this, we can then add that, at the very least, the idealizing assumption of non-interacting particles doesn't impede these core features of explanation in any obvious way, and appears to promote other good-making features of an explanation (e.g. other 
cognitive goods such as easier calculation and flagging irrelevancies). Hence, the derivation is explanatory.

\subsubsection{Constructing New Models}

It is often thought that understanding involves the ability to apply information to novel cases (De Regt 2009, Grimm 2010, Hills 2015). Frequently, idealized bases for one object provide clues as to how to construct idealized bases for another object. For example, we can anticipate that the false assumption of non-interacting particles will be appropriate for dilute gases without knowledge of the details of those interactions, a result that forms a testable prediction of the theory.

The case of liquid helium provides a striking illustration of this process: assuming liquid He-4 to be a non-interacting quantum gas — a clear falsehood, since it is a liquid - predicts that it will transition to a Bose condensate at a temperature of $3.3 \mathrm{~K}$. Experimentally, He-4 undergoes such a transition, becoming a superfluid at $2.17 \mathrm{~K}$. This idealized description of a strongly interacting liquid as a non-interacting gas is not even approximately true, and indeed the transition temperature is off by roughly $50 \%$. Nevertheless, the mere existence of the superfluid phase is an important prediction of the idealized model, and one that a "de-idealized" basis does not entail.

\subsection{Two Improvements}

Using the derivation of the ideal gas law as our example, we have argued that some falsehoods provide understanding. Furthermore, we have offered four different criteria for determining when a proposition provides understanding: simpler calculation, highlighting irrelevancies, explanation, and constructing new models. So long as at least one of these criteria is a mark of a proposition's adequacy in providing understanding, the argument succeeds, though we believe 
that idealizations often strike the "right balance" between these four cognitive goods.

Consequently, the scientific record supports the Provision Condition.

We will now advertise two virtues of the Provision Condition that have been underappreciated in earlier discussions of non-factive understanding: the object-basis distinction, and the corresponding distinction between instrumental and final epistemic value.

\subsubsection{Objects and Bases}

While the distinction between bases and objects may seem obvious, the conflation of these two elements of understanding has led to pernicious confusion between non- and quasi-factivists. For instance, Elgin writes:

The ideal gas law accounts for the behavior of gases by describing the behavior of a gas composed of dimensionless, spherical molecules that are not subject to friction and exhibit no intermolecular attraction. There is no such gas. Indeed, there could be no such gas. Nonetheless, scientists purport to understand the behavior of gases by reference to the ideal gas law (Elgin 2007, 38).

The first and last sentences indicate that Elgin takes the object of understanding to be "the behavior of gases," and the basis to be the ideal gas law. However, the various idealizing assumptions that Elgin associates with the ideal gas law - that gases are "composed of dimensionless, spherical molecules that are not subject to friction and exhibit no intermolecular attraction"- are not part of the ideal gas law. Strictly speaking, the ideal gas law does not describe molecules at all. Rather, it describes the relationship between pressure, volume, temperature, and amount of substance —all of which are macroscopic properties of a gas. The idealizing assumptions associated with the ideal gas law are actually part of the statisticalmechanical model from which the ideal gas law is derived. 
As a result of her presentation of the ideal gas law, some quasi-factivists misinterpret the larger philosophical points she is making. For instance, consistent with the passage from Elgin above, Mizrahi (2012) reads her as treating the ideal gas law as the basis of understanding, and goes on to defend the veracity of the ideal gas law. And to be fair, Elgin's writing suggests this interpretation. But given the idealizations in question, the ideal gas law is best interpreted as the object of understanding, with statistical mechanics serving as its proper basis. Our interpretation is supported by the fact that the understanding-providing fictions in question (e.g. about there being no particle interactions) only figure in the latter.

In fact, Mizrahi (2012) seems to acknowledge this point, but he does not think that these falsehoods support non-factivism. He argues, ...if by saying that the Ideal Gas Law is false, Elgin means that the idealizing assumptions made to derive the law... are wrong, then it doesn't seem to follow that the whole thing [i.e. the ideal gas law] is false. The Ideal Gas Law doesn't give us the truth, the whole truth, and nothing but the truth about the behavior of gases (Mizrahi 2012, 245).

As we have framed the dialectic, this is a red herring. What matters is whether the idealizing assumptions are false. Thus, even if the ideal gas laws provide only some truths about gases (a point that any non-factivist would grant), the question is whether false claims about molecular properties provide us with understanding of the ideal gas law. For reasons we discuss above, this appears altogether plausible. Hence, Mizrahi's dismissal of the importance of statisticalmechanical idealizations for understanding the ideal gas law is too hasty. 


\subsubsection{Epistemic Value}

Many epistemologists take understanding — whether quasi- or non-factive - to be evidence for what is sometimes called epistemic value pluralism, the doctrine that there are multiple epistemic values that are equally fundamental (Elgin 2004, Kvanvig 2005, Pritchard 2010). This stands opposed to epistemic value monism, which holds that there is only one fundamental epistemic value. The dominant form of epistemic value monism is veritism, which holds that true belief is the sole fundamental epistemic good (David 2005, Goldman 1999, Lynch 2009). This means that all other epistemically valuable things are valuable only because they are a means to acquiring true beliefs.

For many reasons, veritism's axiology is thought to be too austere to capture the rich epistemic value of understanding. Let us consider an argument suggesting that non-factive understanding poses a special challenge to veritism:

1. If non-factivism about understanding is true, then some falsehoods are epistemically valuable.

2. If some falsehoods are epistemically valuable, then veritism is false.

3. $\therefore$ If non-factivism about understanding is true, then veritism is false. ${ }^{7}$

The preceding discussion is largely consonant with the first premise: understanding trades in falsehoods that provide cognitive goods. However, the Provision Condition does not support Premise 2. True to its name, the Provision Condition holds that falsehoods "provide" understanding, i.e. falsehoods are a means to understanding, and hence have instrumental value. But, intuitively, we account for the instrumental value of a means by getting a grip on the final

${ }^{7}$ Elgin (2004) offers an argument along these lines. 
value of its end. Hence, veritism is actually consistent with non-factivism about understanding just in case the epistemic value of our four cognitive goods ultimately bottoms out in true belief.

We think that this is precisely the case. For reasons rehearsed in Section 4.1.3, we do not think that explanatory realism should be assumed when discussing scientific explanation, and we also think that the derivation of the ideal gas law satisfies the core features of some prominent theories of explanation. Hence, we think that it is true that the combination of the partition function and the idealizations explains the ideal gas law. So, a semantics for "explains" that is sensitive to scientific practice makes for an easy dance partner with veritism.

Other cognitive goods require even less dancing. Clearly, an idealization only highlights irrelevancies if it points to true claims about what is irrelevant. Similarly, if, as we suggested in Section 4.1.1, easier calculation's aim is to bring the central structures and determinants underlying an object into relief, then those derivational shortcuts better allow us to appreciate true things about those deep structures. Our discussion above shows that the ideal gas law conforms to this basic narrative.

Finally, we can treat our last cognitive good, the construction of new models, as parasitic on the previous three. Presumably, scientists consider the model of liquid helium to be useful because it facilitates calculations, highlights irrelevancies, and explains. As a result, the arguments just rehearsed for the model of the ideal gas law apply to this new model.

So, the Provision Condition allows us to see how non-factivism about understanding is actually compatible with veritism. The key is that the basis-object distinction also maps on to a means-end distinction, which in turn maps on to an instrumental value-final value distinction. Of course, we do not claim to have established veritism - or even to have shown that the epistemic 
value of understanding is exhausted by the epistemic value of true belief. Rather, we only have shown that nothing distinctive about non-factive understanding refutes veritism.

\section{The Parity Condition}

Thus far, we have shown that the derivation of the ideal gas law satisfies the Falsehood and Provision Conditions, i.e. we have argued that some falsehoods sometimes provide understanding. Isn’t this enough to establish non-factivism about understanding? Both nonfactivists and quasi-factivists demur.

In particular, if the Falsehood and Provision Conditions established non-factivism on their own, then understanding could improve as its bases become more accurate. For example, while Copernicus certainly had some understanding of planetary motion, the basis of that understanding (that planetary orbits are circular) is, strictly speaking, false. Hence the Falsehood and Provision Conditions are satisfied. Nevertheless, that basis is both more accurate than its Ptolemaic predecessor, and provides less understanding than its more accurate Keplerian successor. These considerations would suggest that understanding improves in proportion to accuracy, with historical examples showing that this improvement is often gradual. Pretheoretically, this seems broadly quasi-factivist in spirit. Indeed, some quasi-factivists (Mizrahi 2012, 244) claim that idealizations behave in precisely this manner.

The Parity Condition clarifies the sense in which idealizations must not be mere "way stations" (Elgin 2007, 41) to better, more accurate understanding. Recall that this condition requires non-factivists to establish the following:

The understanding of $x$ resulting from either not accepting a falsehood $p$ or accepting a more accurate proposition instead of $p$ is not better than the understanding provided by $\operatorname{accepting} p$. 
The rough idea is that non-factivism is only established if understanding does not always improve as its bases become more accurate. In this way, the Copernicus example fails to satisfy the Parity Condition. Note that non-factivists need only "play for the tie," i.e. falsehoods need not provide better understanding than their more accurate counterparts; it suffices if the understanding afforded by falsehoods is no worse than the understanding provided by a more accurate representation. After all, it would already seem to be a significant result if understanding were sometimes indifferent to accuracy.

We begin by defending the claim that the idealized derivation of the gas law satisfies the Parity Condition. We then advertise two ways in which the Parity Condition is an improvement over other proposals in the non-factive understanding literature.

\subsection{Two (More) Objections Rebutted}

Insofar as there are positive arguments for the claim that the idealized derivation of the ideal gas law satisfies the Parity Condition, they are twofold. First, the derivation is still acceptable to many working scientists. It has not gone the way of Ptolemaic astronomy or other discarded scientific ideas. As we argued in Section 4.1.4, the idealization of non-interacting particles has even had a productive career outside of the ideal gas law. While this is far from a water-tight argument, the fact that scientists have not abandoned this idealization is at least evidence that they find some value in the cognitive goods that it provides.

Second, one of the lemmas in the Parity Condition is clearly on firm ground: if we simply eliminated the idealization of non-interacting particles, then our derivation would stall at the partition function (Eq. 1), as the assumption of non-interacting particles is what allows us to infer that the available phase space for each particle is proportional to volume (Eq. 2), which in turn leads to the ideal gas law (Eq. 3). Three of our cognitive goods - facilitating calculation, 
highlighting irrelevancies, and explanation (covered in Sections 4.1.1-4.1.3 respectively)—hinge on a valid derivation of the ideal gas law from the partition function. The last-constructing new models - hinges on an analogous derivation of a different equation of state (e.g. liquid He-4, as discussed in Section 4.1.4). Hence, simply eliminating this idealization excludes all the understanding characteristic of these cognitive goods.

As a result, quasi-factivism can only be redeemed by showing that replacing this idealization with something more accurate provides greater understanding of the ideal gas law. With this in mind, we will consider and rebut two objections to the claim that the idealization satisfies the Parity Condition.

\subsubsection{The van der Waals Objection}

For quasi-factivists, one tempting move is to claim that understanding improves as more accurate representations replace the ideal gas law (Mizrahi 2012). The leading example offered here is the van der Waals equation:

$$
\text { (Eq. 4) } \quad\left(P V+\frac{a N^{2}}{V^{2}}\right)(V-N b)=N k T
$$

In comparison with the ideal gas law, the two new parameters, $a$ and $b$, represent intermolecular attraction and molecular volume, respectively. As with the ideal gas law, this equation of state can also be derived from statistical mechanics, though unlike the ideal gas law, derivation of the van der Waals equation assumes that particles have volume and interact. The particles' hard cores lead to a decrease in the available volume for each particle proportional to $N$, and the mean field attraction gives an interaction proportional to particle density $N / V$. Both effects thus involve a more complicated dependence on $V$ in the partition function, leading to a modified equation of state. Hence, when compared to the ideal gas law and its attendant statisticalmechanical model, both the object and the basis of understanding are more accurate. 
Furthermore, as Mizrahi $(2012,243)$ notes, the van der Waals equation "explains the deviations from the Ideal Gas Law and it describes the behavior of gases over a wider range of temperatures and pressures." Presumably, these are marks of better understanding. So, if this reasoning is sound, it quickly defeats non-factivism.

However, this argument faces three flaws. First, it is a non sequitur. To see this, observe that the transition from the ideal gas law to the van der Waals equation involves a change in both the basis and the object of understanding. Specifically, the object of understanding has shifted from the ideal gas law (Eq. 3) to the van der Waals equation (Eq. 4). However, this shift is consistent with non-factivism, for given two objects of understanding, $x_{1}$ and $x_{2}$, it is no requirement that a basis for understanding one object, $x_{1}$, should also provide understanding of another object, $x_{2}$. Indeed, without further qualification, this requirement is clearly absurd. For instance, it would require a common basis of understanding for the ideal gas law and the Second World War.

Taken less literally, one might argue that the ideal gas law and the van der Waals equation are so similar that they should have an identical basis. But even this modest requirement is debatable. Consider a simpler example, in which two patients have identical symptoms, save that one breaks out in a mild rash when exposed to temperatures over 75 degrees Fahrenheit. Clearly, these two patients' symptoms are quite similar, yet it is hardly required that the same disease must provide understanding of their symptoms. Hence, there is no mandate that a common basis provide understanding for any two objects, $x_{1}$ and $x_{2}$, even if they are very similar.

Second, even on a charitable recasting of this argument, it runs afoul of scientific practice. Suppose that we did not interpret purveyors of this objection as conflating bases and 
objects, but instead as expanding the quasi-factivist thesis so that understanding improves not only when its bases become more accurate, but also when its objects become more accurate. At first blush, this looks plausible. Moreover, it would allow quasi-factivists to dispense with the ideal gas law by appeal to the van der Waals equation while avoiding the aforementioned non sequitur.

However, this is very far removed from scientific practice. This expanded quasi-factivist doctrine entails that all equations of state are mere way stations for the most accurate equation of state, the virial expansion:

$$
\text { (Eq. 5) } \quad \frac{P V}{N k T}=1+\frac{B}{V}+\frac{C}{V^{2}}+\frac{D}{V^{3}}+\ldots
$$

This expansion can be derived directly from statistical mechanics, and rendered arbitrarily precise by extending the equation indefinitely, with each added term being derivable from increasingly detailed and accurate assumptions about the intermolecular forces. For instance, $B$ corresponds to interactions between pairs of molecules; $C$, triplets; $D$, quartets; etc.

However, the statistical-mechanical model used to derive the virial equation of state is not the only (or even the chief) model used to understand gases. Indeed, since non-factivism only holds that some falsehoods provide equally good understanding as more veridical alternatives, a single example of using a non-virial equation of state suffices. Yet examples abound. For instance, at high enough temperatures and pressures, past what is known as the critical point, a clean distinction between liquid and solid breaks down. For gases at temperatures above the critical temperature, the Redlich-Kwong equation of state,

$$
\text { (Eq. 6) } \quad P=\frac{N k T}{V_{m}-b}-\frac{a}{\sqrt{T} V_{m}\left(V_{m}+b\right)} \text {, }
$$

is often used. Here, $V_{m}=V / N$, while $a$ and $b$ are determined empirically based on the critical point of the gas, rather than derived directly from statistical mechanics. Other equations of state 
with a higher number of empirically determined parameters exist, such as the Benedict-WebbRudin equation of state, with eight. Scientists do not normally take a detour through the virial expansion to arrive at these equations of state. It's far more plausible that each of these equations of state is a distinct object of understanding, rather than each being a way station for the virial expansion. After all, scientists can already derive the virial expansion, so why would they continue to discover other equations of state?

Third, this argument fails to explain why the assumption that particles are non-interacting figures not only in our understanding of ideal gases, but also our understanding of other equations of state as well. For example, above, we discussed how the equation of state for liquid He-4 still assumes non-interacting particles. By contrast, the quasi-factivist argument we are currently considering wrongly suggests that this practice does not advance our understanding.

In sum, we take quasi-factivists' appeals to more accurate equations of state, such as the van der Waals equation, to miss their mark. These arguments do not address the specific challenge that non-factivism raises, conflict with scientists' use of a wide variety of equations of state, and cannot account for all of the ways in which idealizations provide understanding. Hence, this objection leaves our idealization's claim to the Parity Condition unfazed.

\subsubsection{The Virial Objection}

While the van der Waals Objection does not quite hit its mark, it's in the vicinity of a deeper challenge to non-factivism. As noted above, the virial expansion,

$$
\text { (Eq. 5) } \quad \frac{P V}{N k T}=1+\frac{B}{V}+\frac{C}{V^{2}}+\frac{D}{V^{3}}+\ldots,
$$

can be derived directly from statistical mechanics, and can be rendered arbitrarily precise with respect to its representation of particle interactions. Furthermore, the ideal gas law (Eq. 3) can be derived from this expansion (Eq. 5): since the ideal gas law obtains at low density $(P)$ and high 
temperature $(T)$, volume $(V)$ will have to be high in comparison to the relevant distance scales in order for the left-hand side of this equation to differ significantly from 1 . However, high volumes mean that all of the terms on the right-hand side of the virial equation that represent particle interactions, $B / V, C / V^{2}$, etc., will be vanishingly small. The resulting equation is the ideal gas law. Hence, just as with the derivation in Section 3, this derivation results in the ideal gas law, but does so without adverting to the idealization. Furthermore, the derivation tells us why the idealization of non-interacting particles works, so it appears to provide us with deeper, and hence better understanding of the ideal gas law.

If this objection is sound, it would appear to undercut non-factivism. However, the argument faces two difficulties. First, deriving the ideal gas law from the virial expansion also requires an idealization. In particular, to get from the virial expansion (Eq. 5) to the ideal gas law (Eq. 3), volume and number of particles (the gas' extensive quantities) must approach infinity, while their ratios (the gas' intensive properties) are held fixed. Hence, at best, quasi-factivists can only show that one idealization (non-interacting particles) piggybacks off of another (the thermodynamic limit).

In response, quasi-factivists may argue that even though this derivation involves an idealization, it nevertheless trades in a more accurate basis than the derivation of the ideal gas law involving non-interacting particles. However, this merely postpones the inevitable non- 
factivist victory — even if the original idealization of non-interacting particles is a mere way station, there would be no way of eliminating the idealizations in the thermodynamic limit. ${ }^{8}$

Second, it is not at all obvious that this "virial derivation" provides better understanding than the idealized version presented in Section 4.1. Once again, recall our four cognitive goods: explanation, highlighting irrelevancies, easier calculation, and the construction of new models. The virial derivation does better with respect to some of these criteria, and worse with respect to others (see Table 1). Let's begin with what appears, at first blush, to be the greatest strength of the virial derivation - that it is "deeper" because it shows how the idealization works. This is achieved by showing how the added terms in the virial expansion ultimately drop out when density is low and temperature is high. In this regard, it tells us why particle interactions are irrelevant to the behavior of ideal gases. However, it is less clear that it does as well in showing us that particle interactions are irrelevant in this way (Strevens 2016). After all, the irrelevance of particle interactions is made especially salient when the ideal gas law can be derived from the partition function on the assumption that particles do not interact at all. So, each derivation highlights different aspects of particle interactions' irrelevance to the ideal gas law. It is, for all practical purposes, a wash on this front.

Similarly, it is hard to see why the idealized derivation amounts to an inferior explanation of the ideal gas law than its virial counterpart (Section 4.1.1). Quasi-factivists cannot simply assume that accurate explanations necessarily provide better understanding without begging the

\footnotetext{
${ }^{8}$ A more promising quasi-factivist route is to deny that this use of the thermodynamic limit is an idealization at all, as Norton (2012) does. Whether these arguments succeed, or fall short, as Batterman (2013) argues, exceeds this paper's scope.
} 
question. Parallel points apply to the construction of new models (Section 4.1.4). Moreover, since the aforementioned models were first constructed with idealizations, and only subsequently de-idealized, it is unclear whether the virial expansion fares as well on this front. Finally, the virial derivation clearly requires more involved calculations (Section 4.1.2), so in this regard, it fares worse than its idealized counterpart.

\begin{tabular}{|c|c|c|}
\hline $\begin{array}{c}\text { Ways of Providing } \\
\text { Understanding }\end{array}$ & $\begin{array}{c}\text { 'Idealized' Derivation } \\
\text { (Assumes No Interactions) }\end{array}$ & $\begin{array}{c}\text { 'De-Idealized' Derivation } \\
\text { (Virial Expansion) }\end{array}$ \\
\hline $\begin{array}{c}\text { Facilitates Calculation? } \\
\text { (Section 4.1.1) }\end{array}$ & $\begin{array}{c}\text { Comparatively better } \\
\text { Comparatively worse }\end{array}$ \\
\hline $\begin{array}{c}\text { Highlights the Irrelevance of } \\
\text { Particle Interactions? } \\
\text { (Section 4.1.2) }\end{array}$ & $\begin{array}{c}\text { More clearly shows that } \\
\text { interactions are irrelevant }\end{array}$ & $\begin{array}{c}\text { More clearly shows why } \\
\text { interactions are irrelevant } \\
\text { (how the idealization works) }\end{array}$ \\
\hline $\begin{array}{c}\text { Explains the Ideal Gas Law? } \\
\text { (Section 4.1.3) }\end{array}$ & Yes & Yes \\
\hline $\begin{array}{c}\text { Facilitates Construction of } \\
\text { New Models? } \\
\text { (Section 4.1.4) }\end{array}$ & Yes & $\begin{array}{c}\text { Yes, though history of } \\
\text { modeling favors idealizations }\end{array}$ \\
\hline
\end{tabular}

Table 1: Relative strengths and weaknesses of idealizations in providing understanding

Thus, the verdict is decidedly mixed, if not slightly favorable to non-factivism, with respect to these four marks of understanding. Undoubtedly, in some contexts, inquirers place greater weight on those criteria with respect to which the idealized derivation surpasses its de-idealized counterpart. These cases support the Parity Condition.

\subsection{Two (More) Improvements}

Using the derivation of the ideal gas law as our example, we have argued that some falsehoods provide understanding in a way that is not a mere way station to better, more accurate understanding. We have thus shown that the example of the ideal gas law satisfies the Parity Condition, and we have completed our case for non-factivism about understanding. As we did with the Provision Condition, we discuss two comparative advantages of the Parity Condition over some previous ways of discussing non-factive understanding. 


\subsubsection{Belief and Acceptance}

The Parity Condition is a constraint on what putative understanders accept, rather than what they believe (Cohen 1992). While different theorists draw that distinction in different ways, for our purposes, the relevant difference is that it is sometimes rational to accept propositions known to be false if including these propositions among one's premises serves certain context-specific purposes, while it is never rational to believe propositions known to be false. The assumption of non-interacting particles ably fits the bill: scientists know it to be false, but it is included as a premise in a derivation, and that derivation's "purpose" can then be spelled out in terms of our four cognitive goods.

To be sure, we are not the first to introduce the belief-acceptance distinction in discussions of non-factive understanding; Elgin (2004) also does so. ${ }^{9}$ However, she introduces the distinction without the detailed relationships specified in the Parity Condition. Without these details, it becomes less clear why understanders should accept false propositions rather than believe closely related, but suitably hedged, true propositions. Indeed, this lacuna in Elgin's account of acceptance may well be the motivation behind the Simple Approximation Objection in Section 3.1.2. By contrast, Section 5.1.2 shows how attempts to accept an approximate truth in

${ }^{9}$ Elgin has been misinterpreted on this point. For instance, in a widely read survey of the literature on epistemic value, Pritchard (2007) takes Elgin to assert that in the case of idealizations, "scientists... have false beliefs in the subject matter." He then goes on to suggest that "scientists might well accept their theories in such cases (i.e. endorse them for all practical purposes, as the best theory available), even though they don't actually believe them." Curiously, this comes three years after Elgin made precisely the same distinction! 
place of the idealization do not result in better understanding-precisely as the Parity Condition requires.

Our belief-acceptance distinction also helps to clarify just who is a non-factivist. Perhaps the best advertisement for its utility on this front is Strevens' (2008, 2013, 2016) nuanced discussions of understanding and idealization. Strevens reaches very similar verdicts as ours about the benefits of idealization to understanding. The differences between our views are twofold. First, we delve a bit more into the relevant derivations than Strevens, which allows us to make kindred points with a bit more precision. We consider this difference to be minor, and bracket it hereafter.

The second and more important difference is that Strevens largely bypasses explicit discussion of quasi- and non-factive understanding, so his stance on this issue is not altogether clear. The Parity Condition clearly puts Strevens in the non-factivist camp. For instance, he rebuts arguments showing that adverting exclusively to the truth improves understanding (Strevens 2016, 37).

He also points out that idealized explanatory models do no worse than "canonical" models, i.e. models containing nothing but truths about difference-makers. Indeed, idealized models sometimes do better in enabling scientists to "grasp the reasons things do and do not make a difference to a phenomenon" (Strevens 2016, 48). All of this favors a non-factivist interpretation of Strevens.

What, then, are we to make of certain "Strevensian" remarks that suggest a quasi-factivist reading? For example,

I hold that idealizations that appear to make some false assumption about the world — for example, the assumption that there are no long-range intermolecular forces (in the ideal 
gas model) ...-in fact make true claims about difference-making. When used to explain the approximate truth of Boyle's law, for example, the ideal gas model, when it sets longrange intermolecular forces to zero, is in fact saying, when properly interpreted, that long-range intermolecular forces make no difference to the law's approximately holding (Strevens 2013, 512).

In the current discussion, we can take Strevens' non-factivist moments to counsel what we are entitled to accept for the purposes of understanding the ideal gas law - that particles in an ideal gas do not interact. Indeed, this makes the appeal to acceptance fit naturally with our earlier remark that different marks of understanding will carry greater weight in different contexts. For instance, for the purposes of understanding the ideal gas law by means of easy calculation and in order to highlight that particles interactions make no difference, we are entitled to accept the idealization. All of this is compatible with Strevens' more quasi-factivist remarks counseling us on what we ought to believe - namely that particle interactions do not make a difference to the ideal gas law. Without the belief-acceptance distinction, Strevens' stance on the factivity of understanding is unclear. Hence, the Parity Condition can help us understand exactly where different interlocutors' arguments fall in the factivism debate.

\subsubsection{Center and Periphery}

A second advantage to the Parity Condition concerns quasi- and non-factivists' different responses to false bases. The Parity Condition counsels that they ought to differ about whether eliminating or replacing those falsehoods would result in better understanding. This stands opposed to the more prominent distinction in the literature between "central" and "peripheral" propositions in a person's understanding. We shall argue that the Parity Condition outperforms 
the central-peripheral distinction in identifying the falsehoods over which quasi- and nonfactivists should be debating.

According to quasi-factivists, peripheral propositions can admit falsehoods without undermining understanding, but central propositions must be true if one is to understand (Kvanvig 2003, 2009a, Mizrahi 2012). However, as Kvanvig (2009a, 341) admits, "there is vagueness in the distinction between central and peripheral pieces of information. When falsehoods are found within the noetic system of a particular person, it will often be vague whether that falsehood is sufficient to undermine understanding." This vagueness is precisely what worries us, as it makes quasi-factivism all but unfalsifiable, since any inconvenient idealization can be too easily dismissed as merely peripheral.

Furthermore, the few principled central-peripheral distinctions in the literature are flawed. For example, some claim that clearly specified boundary conditions allow us to identify central propositions (Mizrahi 2012, 251). On this view, a model's peripheral idealizations are either discarded or lose their idealized status and become (approximately) true once scientists determine appropriate boundary conditions for that model. These boundary conditions are set by establishing when the model holds true. In the case of the ideal gas law, this occurs under relatively low pressure and high temperature. ${ }^{10}$ However, this reasoning fails to undermine nonfactivism. Presumably, it depends on the following:

(BC1) If $p$ 's boundary conditions are adequately specified and $p$ provides understanding of $x$, then $p$ is central to understanding $x$.

${ }^{10}$ Once again, recall that Mizrahi conflates bases and objects, see Section 4.2.1. 
It is unclear why this is true. If $p$ 's boundary conditions are so narrow that $p$ is barely applicable to $x$-related matters, $p$ appears "peripheral" to understanding of $x$.

More damning, however, is that $\mathrm{BC} 1$ cannot establish that understanding is quasi-factive. While both our critique of simple approximation views (Section 3.1.2) and our discussion of the thermodynamic limit (Section 5.1.2) suggest otherwise, let us grant the following for the sake of argument:

(BC2) If $p$ 's adequately specified boundary conditions are $c$, then $p$ is approximately true in $c$.

Combined, BC1 and BC2 entail:

(BC3) If $p$ 's boundary conditions $c$ are adequately specified and $p$ provides understanding of $x$, then $p$ is central to understanding $x$ and is approximately true in $c$.

However, non-factivists only need some central propositions to be false, and BC3 is consistent with there being a false but central proposition with unspecified boundary conditions. Hence, $\mathrm{BC} 1$ and $\mathrm{BC} 2$ are not only objectionable; they don't even support the quasi-factivist's desired conclusion.

Perhaps these difficulties are surmountable. Until then, quasi-factivists should be worried that a proposition's "centrality" amounts to little more than "whatever is required to make it (approximately) true." That, of course, would be circular. By contrast, the Parity Condition provides a comparatively precise and non-question-begging criterion of when a falsehood supports quasi-factivism: when either omitting it or replacing it with something more accurate results in an increase of cognitive goods. Consequently, the Parity Condition is a more serviceable yardstick for adjudicating between non- and quasi-factivism than the central- 
peripheral distinctions that have been offered thus far.

\section{Conclusion}

We have argued that non-factivism about understanding is a defensible doctrine. Along the way, we have appealed to both more accurate (e.g. in Section 4.2.1), and more precise (e.g. in Sections 3, 4.1, and 5.1) descriptions of the science behind the ideal gas law to assist our philosophical arguments. In this way, we have sought to be conscientious naturalists.

We do not regard this naturalism as at odds with any of its traditional philosophical foils. Begin with a standard opposition between naturalistic and "traditional" epistemology. The difference frequently boils down to methodology: do we argue for an epistemological thesis by appeal to science or by using the more shopworn epistemological tools of conceptual analysis, thought experiments, etc.? While we have not needed any thought experiments, we have regimented and policed various conceptual distinctions, like good analytic epistemologists. In particular, we have shown how failure to make several conceptual distinctions-between basis and object (Section 4.2.1), instrumental and final value (Section 4.2.2), and belief and acceptance (Section 5.2.1) —as well as an overzealousness to make others — between center and periphery (Section 5.2.2) —have hindered earlier discussions of non-factive understanding. So, a proper philosophical account of understanding ought to draw liberally from the toolboxes of both naturalistic and traditional epistemology.

Another distinction is between the descriptive and the normative, and here, of course, naturalism has a complex history. Nevertheless, our arguments, which make ample appeal to descriptions of science, suggest several normative claims:

(1) It is sometimes rational to accept a falsehood in the context of understanding. 
(2) Ease of calculation, the highlighting of irrelevancies, explanation, and the construction of new models are cognitive goods.

(3) Non-factivism about understanding is compatible with veritism about epistemic value.

(4) The epistemic norms governing acceptance differ from those for belief.

As the italicized claims make clear, naturalists need not neglect normativity. Indeed, if our arguments are sound, then unlike the particles of an ideal gas, the lack of interaction between naturalism and normativity is a falsehood that has hampered our understanding.

Batterman, Robert W. 2000. "A 'modern' (= Victorian?) attitude towards scientific understanding." Monist 83 (2):228-257.

Batterman, Robert W. 2002. The devil in the details: asymptotic reasoning in explanation, reduction and emergence. Oxford: Oxford University Press.

Batterman, Robert W. 2013. "The Tyrrany of Scales." In The Oxford handbook of philosophy of physics, edited by Robert W. Batterman, 255-286. New York: Oxford University Press.

Braverman, Mike, John Clevenger, Ian Harmon, Andrew Higgins, Zachary Horne, Joseph Spino, and Jonathan Waskan. 2012. "Intelligibility is necessary for scientific explanation, but accuracy may not be." In Proceedings of the thirty-fourth annual conference of the cognitive science society, edited by Naomi Miyake, David Peebles and Richard Cooper. Cognitive Science Society.

Cohen, L. Jonathan. 1992. An essay on belief and acceptance. Oxford: Clarendon Press.

David, Marian. 2005. "Truth as the primary epistemic goal: A working hypothesis." In Contemporary debates in epistemology, edited by M. Steup, J. Turri and E. Sosa, 363377. Malden: John Wiley \& Sons.

De Regt, Henk W. 2009. "Understanding and scientific explanation." In Scientific understanding: philosophical perspectives, edited by Henk W. De Regt, Sabina Leonelli and Kai Eigner, 21-42. Pittsburgh: University of Pittsburgh Press.

De Regt, Henk W., and Dennis Dieks. 2005. "A contextual approach to scientific understanding." Synthese 144 (1):137-170.

Elgin, Catherine Z. 2004. "True enough." Philosophical issues 14 (1):113-131. doi: 10.1111/j.1533-6077.2004.00023.x.

Elgin, Catherine Z. 2007. "Understanding and the facts." Philosophical studies 132 (1):33-42.

Elgin, Catherine Z. 2009a. "Exemplification, idealization, and scientific understanding." In Fictions in science: philosophical essays on modeling and idealization, edited by Mauricio Suárez, 77-91. London: Routledge.

Elgin, Catherine Z. 2009b. "Is understanding factive?" In Epistemic value, edited by Adrian Haddock, Allan Millar and Duncan Pritchard, 322-330. Oxford: Oxford University Press. 
Goldman, Alvin I. 1999. Knowledge in a social world. Cambridge: Oxford University Press.

Greco, John. 2013. "Episteme: knowledge and understanding." In Virtues and their vices, edited by Kevin Timpe and Craig A. Boyd, 285-301. Oxford: Oxford University Press.

Grimm, Stephen R. 2010. "The goal of understanding." Studies in the history and philosophy of science 41 (4):337-344. doi: 10.1016/j.shpsa.2010.10.006.

Hills, Alison. 2015. "Understanding why." Noûs 49 (2):661-688. doi: 10.1111/nous. 12092.

Khalifa, Kareem. 2012. "Inaugurating understanding or repackaging explanation?" Philosophy of science 79 (1):15-37.

Kvanvig, Jonathan L. 2003. The value of knowledge and the pursuit of understanding. Cambridge: Cambridge University Press.

Kvanvig, Jonathan L. 2005. "Truth is not the primary epistemic goal." In Contemporary debates in epistemology, edited by M. Steup, J. Turri and E. Sosa, 352-362. Malden: John Wiley $\&$ Sons.

Kvanvig, Jonathan L. 2009. "Responses to critics." In Epistemic value, edited by Adrian Haddock, Allan Millar and Duncan Pritchard, 339-352. Oxford: Oxford University Press.

Kvanvig, Jonathan L. 2009a. "Précis of the value of knowledge and the pursuit of understanding." In Epistemic value, edited by A. Haddock, A. Millar and D. Pritchard, 309-313. Oxford: Oxford University Press.

Lewis, David K. 1986. "Causal explanation." In Philosophical papers, 214-240. Oxford: Oxford University Press.

Lipton, Peter. 2009. "Understanding without explanation." In Scientific understanding: philosophical perspectives, edited by Henk W. De Regt, Sabina Leonelli and Kai Eigner, 43-63. Pittsburgh: University of Pittsburgh Press.

Lynch, Michael P. 2009. "The values of truth and the truth of values." In Epistemic value, edited by Adrian Haddock, Allan Millar and Duncan Pritchard, 225-242. Oxford: Oxford University Press.

Mizrahi, Moti. 2012. "Idealizations and scientific understanding." Philosophical studies 160 (2):237-252. doi: 10.1007/s11098-011-9716-3.

Norton, John D. 2012. "Approximation and Idealization: Why the Difference Matters*." Philosophy of Science 79 (2):207-232. doi: 10.1086/664746.

Oddie, Graham. 2016. "Truthlikeness " In The Stanford Encyclopedia of Philosophy, edited by Edward N. Zalta. URL = $<$ https://plato.stanford.edu/archives/win2016/entries/truthlikeness/>.

Pritchard, Duncan. 2007. "Recent work on epistemic value." American philosophical quarterly $44(2): 85$.

Pritchard, Duncan. 2010. "Knowledge and understanding." In The nature and value of knowledge: three investigations, edited by D. Pritchard, A. Millar and A. Haddock, 3-90. Oxford: Oxford University Press.

Rancourt, Benjamin T. forthcoming. "Better Understanding Through Falsehood." Pacific Philosophical Quarterly. doi: 10.1111/papq.12134.

Strevens, Michael. 2008. Depth: an account of scientific explanation. Cambridge: Harvard University Press.

Strevens, Michael. 2013. "No understanding without explanation." Studies in history and philosophy of science part A 44 (3):510-515. doi: 10.1016/j.shpsa.2012.12.005. 
Strevens, Michael. 2016. "How idealizations provide understanding." In Explaining understanding: new essays in epistemology and philosophy of science, edited by Stephen Grimm, Christoph Baumberger and Sabine Ammon, 37-49. New York: Routledge.

Wilkenfeld, Daniel A., and Jennifer K. Hellmann. 2014. "Understanding beyond grasping propositions: A discussion of chess and fish." Studies in history and philosophy of science part $A 48$ (0):46-51. doi: 10.1016/j.shpsa.2014.09.003.

Woodward, James. 2003. Making things happen: a theory of causal explanation. New York: Oxford University Press. 